\title{
The in vitro cytotoxicitiy of silica nanoparticles at high concentrations strongly depends on the metabolic activity type of cell line
}

Jeng-Sheng Chang, Ke Liang B. Chang, Deng-Fwu Hwang, Zwe-Ling Kong*

Department of Food Science, National Taiwan Ocean University, 2 Pei-Ning Road, Keelung 20224, Taiwan, R.O.C.

\section{Supporting Information}

* To whom correspondence should be addressed:

Zwe-Ling Kong

Department of Food Science

National Taiwan Ocean University

2 Pei-Ning Road, Keelung 20224

Taiwan, R.O.C.

phone: +886224622192 ext. 5130

fax: $\quad+886224632179$

e-mail: kongzl@mail.ntou.edu.tw

* To whom correspondence should be addressed:

Corresponding author Tel.: +886-2-24622192 ext. 5130; fax: +886-2-24632179.

E-mail address: kongzl@ mail.ntou.edu.tw (Zwe-Ling Kong) 


\section{Materials and Methods.}

\section{Nanoparticle preparation and dispersion.}

Sodium silicate was dissolved in $30 \mathrm{~mL}$ buffer (0.05M Sodium acetate) to prepare $1 \%(\mathrm{w} / \mathrm{w})$ solution $(\mathrm{pH}=7.0)$. Immediately after been dissolved into the buffer solution on a magnetic stirrer, it was moved from the stirrer to the bench top. The solution was left to react under ambient condition at ca. $25^{\circ} \mathrm{C}$ for $24 \mathrm{~h}$ before centrifugation to retrieve the silica nanoparticles (silicate).

Silica nanoparticles were also prepared by base-catalyzed sol-gel reaction of TEOS using the seeded-growth technique (1). For the synthesis of nanoparticles, $9 \mathrm{~mL}$ of TEOS, $10.2 \mathrm{~mL}$ of ammonia (25 wt \%), $6 \mathrm{~mL}$ of deionized water, and $300 \mathrm{~mL}$ of ethanol were introduced into a 500-mL glass flask. After the mixture was stirred at $50^{\circ} \mathrm{C}$ for $3 \mathrm{~h}$, ethanol was removed from the system by heating in a rotary-evaporator. The concentrated solution was repeatedly refilled with deionized water for ca. 10 times before centrifugation to obtain the silica nanoparticles (TEOS).

Chitosan samples purchased from a commercial supplier were analyzed for the degree of deacetylation (DD) and molecular weight (MW) (2). The DDs of chitosan samples were $81 \%$ with an MW of $200 \mathrm{kDa}$. Chitosan-silica nanoparticles were prepared by sodium silicate and chitosan solution (3). Sodium silicate was dissolved in $30 \mathrm{~mL}$ buffer ( $0.05 \mathrm{M}$ Sodium acetate) to prepare $0.82 \%(\mathrm{w} / \mathrm{w})$ solution. Immediately 
after the dissolution of silicate on a magnetic stirrer, $3 \mathrm{~mL}$ chitosan solution $(0.28 \%$ w/w) was added. The solution containing silicate and chitosan was mixed completely. Afterwards, it was moved from the stirrer to the bench top. Chitosan- silica nanoparticles were centrifuged after 15 to $360 \mathrm{~min}$ of synthesis. The silica-chitosan nanoparticles prepared after different hours of reaction will be designated as silica-chitosan 1H-6H (nanoparticles).

\section{Nanoparticle characterization.}

Laser light scattering was performed to measure the size of nanoparticle with a Malvern 4700c submicron particle analyzer (Malvern Instruments, Malvern, Worcestershire, UK). Silica or chitosan-silica nanoparticles were suspended in $15 \mathrm{ml}$ deionized water by sonication, further to filtrate with $0.22 \mu \mathrm{m}$ membranes. Each $2 \mathrm{~mL}$ sample was placed in a glass tube and measured for the intensity-averaged particle diameter. Hitachi S-4800 scanning electron microscope (SEM) was used to observe the change in particle size and morphology of nanoparticles. The chemical states of elements in the film were analyzed by an x-ray photoelectron (4) spectroscopy (XPS, VG Scientific ESCALAB 250, the United Kingdom).

\section{Cell culture and exposure to nanoparticles.}


CCD-966sk (human skin fibroblast), WS1 (human normal fibroblast), MRC-5 (human, lung, embryonic), A549 (human, Caucasian, lung, carcinoma), HT-29 (human colon carcinoma), MKN-28 (human gastric adenocarinoma) cell lines came from the American Type Culture Collection (ATCC) and Dr. Murakami’s Research Laboratory (Kyushu University). CCD-966sk (human skin fibroblast), WS1 (human normal fibroblast), MRC-5 (human, lung, embryonic), A549 (human, Caucasian, lung, carcinoma), HT-29 (human colon carcinoma) cell lines were cultured and maintained in Dulbecco's modified eagle's medium (DMEM). MKN-28 (human gastric adenocarcinoma) cell line was cultured and maintained in RPMI 1640 medium supplemented with $10 \%$ fetal bovine serum (FBS). All the cell lines were cultured at $37^{\circ} \mathrm{C}$ in a humidified $95 \%$ air $/ 5 \% \mathrm{CO}_{2}$ environment by seeding into 96 -well plates at the density of 200,000 cells/well in the media. Different concentrations of particles were suspended in deionized water and ultrasonicated for $1 \mathrm{~h}$ to prevent agglomeration. After plating cells for $8 \mathrm{~h}$, the medium with $10 \%$ FBS was removed and replaced by $20 \mu \mathrm{L}$ of particle suspensions at different concentrations. Controls were cells treated with an equivalent volume of serum-free medium without any particles. Cells were incubated for another $48 \mathrm{~h}$ with test particles before the cell viability assay was performed.

Cytotoxicity was assessed using an MTT (3-(4, 5-dimethylthiazol-2-yl)-2, 
5-diphenyltetrazolium bromide) test to measure the succinate dehydrogenase mitochondrial activity (5). Fresh media with $100 \mu \mathrm{L}$ of $5 \mathrm{mg} / \mathrm{mL}$ MTT stain (Sigma-Aldrich in vitro toxicology assay kit) were added into each well and incubated for $4 \mathrm{~h}$. The media/stain was aspirated and the purple color crystals were dissolved with acidic isopropyl alcohol. The absorbance in each well was measured at $570 \mathrm{~nm}$ in BIO-Tek's PowerWave $\mathrm{x}_{\mathrm{x}}$ and $\mu$ Quant microplate spectrophotometers. Background absorbance was measured in a medium containing net nanoparticles (without cells) and net cells (without nanoparticles). All experiments were repeated 3 times to ensure reproducibility. Statistical analysis was carried out using one-way analysis of variance (one-way ANOVA) and Duncan's Test with a level of 95\% significance. The survival rate of the treated cells was calculated in regard to untreated cultures. The relative viability $(\%)$ related to cultures without exposure to nanoparticles was calculated by

$$
\left[\mathrm{A}_{\text {sample }}\right] /[\mathrm{A} \text { control }] \times 100
$$

Where $[\mathrm{A}]_{\text {sample }}$ and $[\mathrm{A}]_{\text {control }}$ denote the absorbance of the sample and control, respectively. All experiments were run in triplicate.

The release of lactate dehydrogenase (6) (LDH) from cultured cells was also monitored over the silica or chitosan modified silica nanoparticles at the concentration range described above. (Cells (CCD, WS1, MRC-5, A549, MKN-28 and HT-29) were seeded in 96-well plates, exposed to silica or chitosan-silica composite nanoparticles, 
and incubated for $48 \mathrm{~h}$.) After $48 \mathrm{~h}$, cultured cells in the 96-well plates were centrifuged at $430 \times \mathrm{g}$ for $5 \mathrm{~min}$ and the cell media was transferred to new 96 -well plates $(50 \mu \mathrm{L} /$ well). Upon addition of $\mathrm{LDH}$ solution $(50 \mu \mathrm{L} /$ well $)$, the media was shielded from the light for $30 \mathrm{~min}$. During this inoculation time, NAD is reduced to $\mathrm{NADH}$ by the $\mathrm{LDH}$ released into the medium. $1 \mathrm{~N} \mathrm{HCl}(25 \mu \mathrm{L} /$ well $)$ was then added to each sample to terminate the reduction of NADH. The resulting absorbance was measured at $490 \mathrm{~nm}$. All experiments were repeated 3 times to ensure reproducibility. Statistical analysis was carried out using one-way analysis of variance (one-way ANOVA) and Duncan's Test with a level of 95\% significance. Control experiments were performed with $0.1 \%$ (w/v) Triton X-100 and set as $100 \%$ cytotoxicity. LDH release was calculated by the following equation:

LDH release $(\%)=\left\{\square[\mathrm{A}]_{\text {sample }}-[\mathrm{A}]_{\text {medium }} \square / \square[\mathrm{A}]_{100 \%}-[\mathrm{A}]_{\text {medium }} \square\right\} \times 100$

Where $[\mathrm{A}]_{\text {sample }},[\mathrm{A}]_{\text {medium }},[\mathrm{A}]_{100 \%}$ denote the absorbance of the sample, medium control and Triton X-100 control, respectively. All experiments were run in triplicate.

\section{X-ray photoelectron spectroscopy (XPS) data.}

To measure the interaction between chitosan and silica, x-ray photoelectron spectroscopy (4) (XPS) is applied to analyze nanoparticles made of silica, chitosan, and silica-chitosan composite. Figure 3 represents the Si $2 p$ binding energies (eV) XPS 
valence-band spectrum for silica, chitosan, and silica-chitosan nanoparticles after $\mathrm{Ar}^{+}$ sputtering. Chitosan nanoparticles do not have the absorption spectrum characteristic of Si $2 p$ binding. The Si $2 p$ peak in silica nanoparticles appears at $102.8 \mathrm{eV}$ suggesting that silicate was present. As for silica-chitosan composite nanoparticles, the binding energies of Si $2 p$ shift to $101.2 \mathrm{eV}$ and the intensity increases dramatically. This indicates Si may have been closely attracted to the amino group in chitosan structure. These XPS spectra suggest that chitosan molecules might attract silicic acids or silanol residue of silica in the solution through ionic interaction with its protonated amino groups, $\mathrm{H}_{3} \mathrm{~N}^{+}$to form silica-chitosan composite nanoparticles (l).

\section{References}

(1) Wang, C.; Zhang, Y.; Dong, L.; Fu, L.; Bai, Y.; Li, T.; Xu, j.; Wei, Y.

Two-dimensional ordered arrays of silica nanoparticles. Chem. Mater. 2000, 12, $3662-3666$.

(2) Chang, K. L. B.; Tai, M. C.; Cheng, F. H. Kinetics and products of the degradation of chitosan by hydrogen peroxide. J. Agric. Food Chem. 2001, 49, 4845-4851.

(3) Chang, J. S.; Kong, Z. L.; Hwang, D. F.; Chang, K. L. B. Chitosan-catalyzed aggregation during the biomimetic synthesis of silica nanoparticles. Chem. Mater. 2006, 18, 702-707.

(4) Handbook of x-ray Photoelectron Spectroscopy, Published by Perkin- Elmer 
Corporation Physical Electronics Division, Minnesota 55344 United States of America.

(5) Mosmann, T. Rapid colorimetric assay for cellular growth and survival: application to proliferation and cytotoxicity assays. J. Immunol. Methods 1983, 65, 55-63.

(6) Promega, CytoTox 96R non-raduoactive cytotoxicity assay technical bulletin, Part\# TB163, revised 9/04. Promega Corporation, Madison, WI 53711-5399 United States of America. 
Table S1. List of binding energies (eV) of XPS valence band-spectrum for silica, chitosan and silica-chitosan nanoparticles before $\mathrm{Ar}^{+}$sputtering.

\begin{tabular}{cccc}
\hline & \multicolumn{3}{c}{ Composition } \\
\cline { 2 - 4 } Elements & chitosan & silica & Silica-chitosan \\
\hline C $1 s$ & 284.6 & 284.7 & 284.4 \\
N $1 s$ & 395.2 & $\mathrm{X}$ & 399.6 \\
$\mathrm{O} 1 s$ & 532.45 & 531.2 & 531.6 \\
$\mathrm{Si} 2 p$ & $\mathrm{X}$ & 102.55 & 102.95 \\
\hline
\end{tabular}


Table S2. List of binding energies (eV) of XPS valence band-spectrum for silica, chitosan and silica-chitosan nanoparticles after $\mathrm{Ar}^{+}$sputtering.

\begin{tabular}{cccc}
\hline & \multicolumn{3}{c}{ Composition } \\
\cline { 2 - 4 } Elements & chitosan & silica & Silica-chitosan \\
\hline C $1 s$ & 284.25 & 284.3 & 284.5 \\
$\mathrm{~N} 1 s$ & 399 & $\mathrm{X}$ & 396.85 \\
$\mathrm{O} 1 s$ & 530.2 & 531 & 532.7 \\
$\mathrm{Si} 2 p$ & $\mathrm{X}$ & 102.6 & 101.8 \\
\hline
\end{tabular}



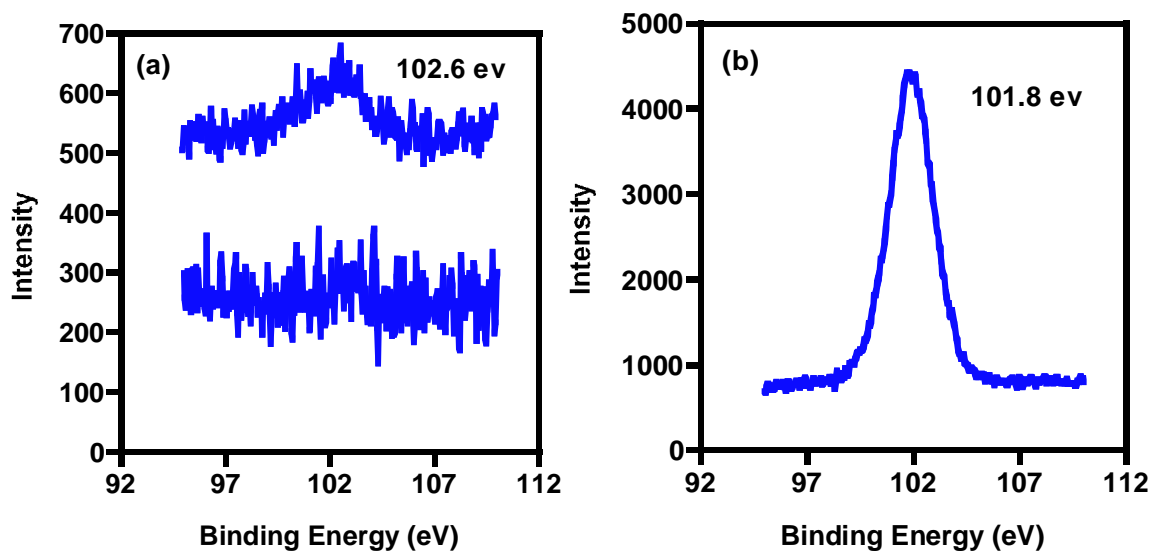

Figure S1. XPS valence band Si 2p spectra of (a) silica nanoparticles (top) and chitosan nanoparticles (bottom), (b) silica-chitosan composite nanoparticles (360 min reaction time). 

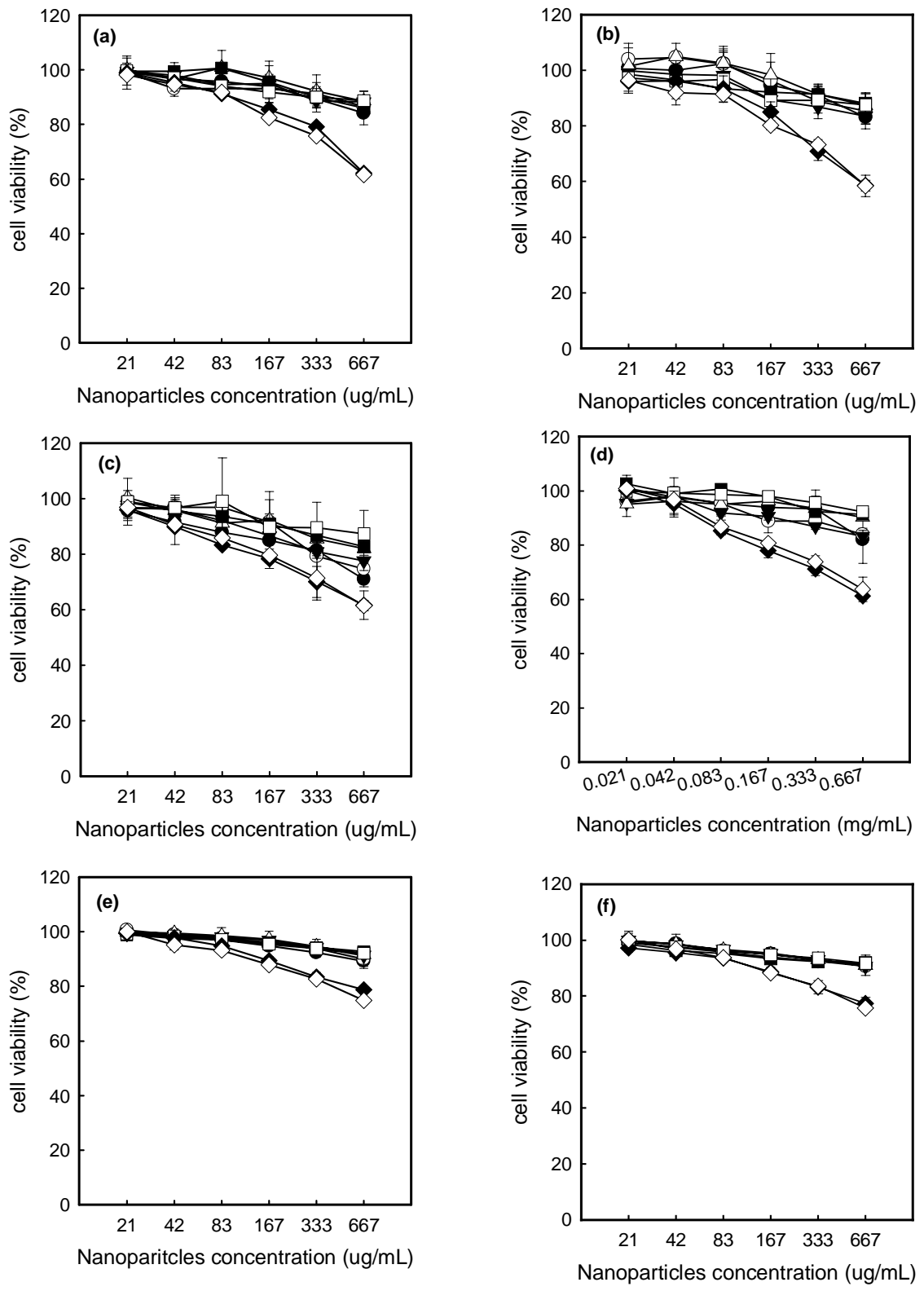

Figure S2. Effect of silica and silica-chitosan composite nanoparticles on the cell viabilities of different cell lines (MTT-assay). (a) CCD, (b) WS1, (c) MRC-5, (d) A549, (e) MKN-28, (f) HT-29. (•) silica-chitosan $1 \mathrm{H}$, ( o) silica-chitosan $2 \mathrm{H}$, ( $\mathbf{\nabla}$ )

silica-chitosan $3 \mathrm{H},(\square)$ silica-chitosan $4 \mathrm{H}$, (ם) silica-chitosan $5 \mathrm{H}$, ( $\square$ ) silica-chitosan $6 \mathrm{H},(\square)$ silica (silicate), ( $\square)$ silica (TEOS). 

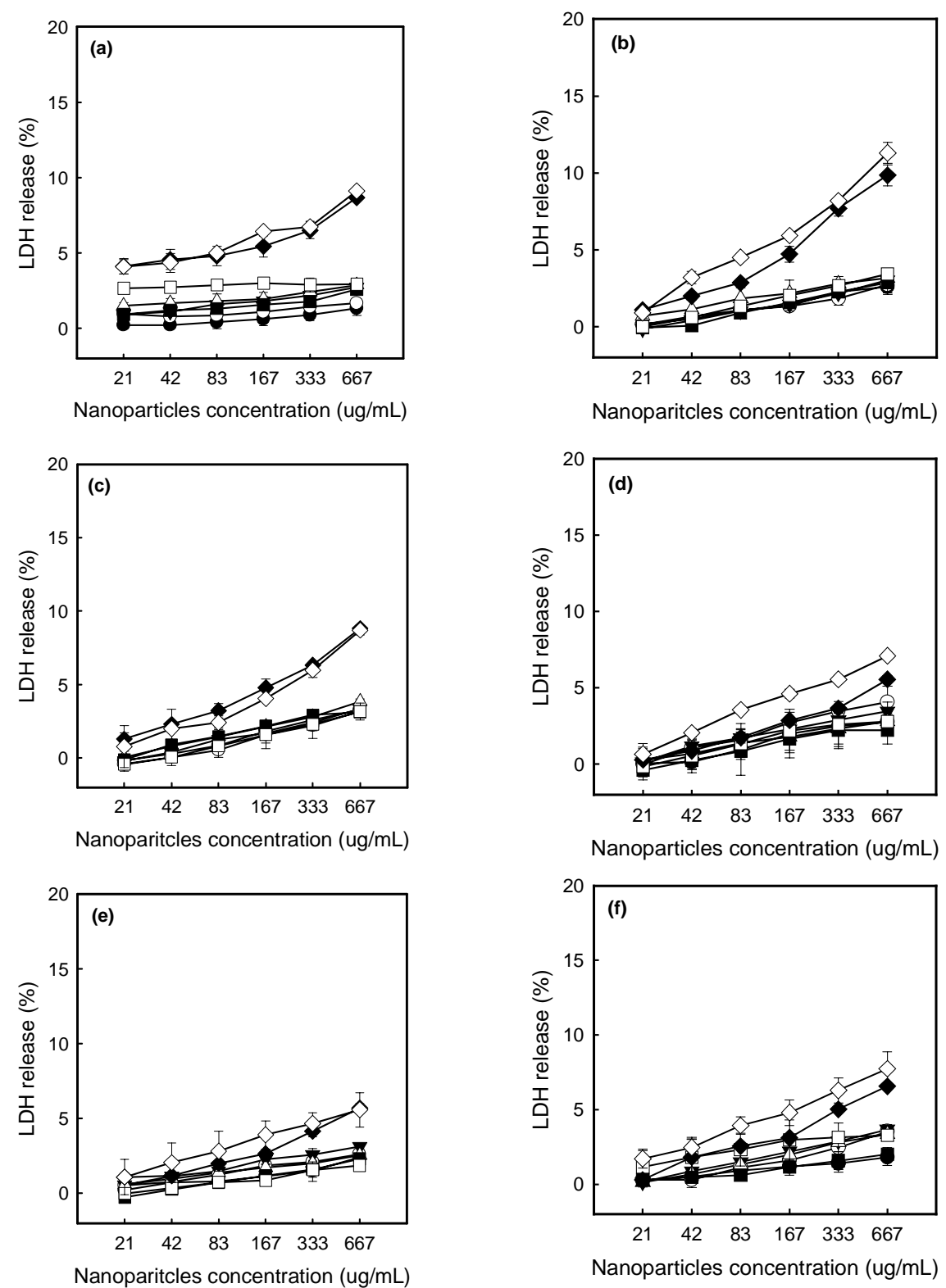

Figure S3. Effect of silica and silica-chitosan composite nanoparticles on the membrane damage of different cell lines (LDH-assay). (a) CCD, (b) WS1, (c) MRC-5, (d) A549, (e) MKN-28, (f) HT-29. (•) silica-chitosan 1 H, ( ०) silica-chitosan 2 H, ( $)$ silica-chitosan $3 \mathrm{H}$, ( $\square$ ) silica-chitosan $4 \mathrm{H}$, (匹) silica-chitosan $5 \mathrm{H},(\square)$ silica-chitosan $6 \mathrm{H},(\square)$ silica (silicate), ( $\square$ ) silica (TEOS). 


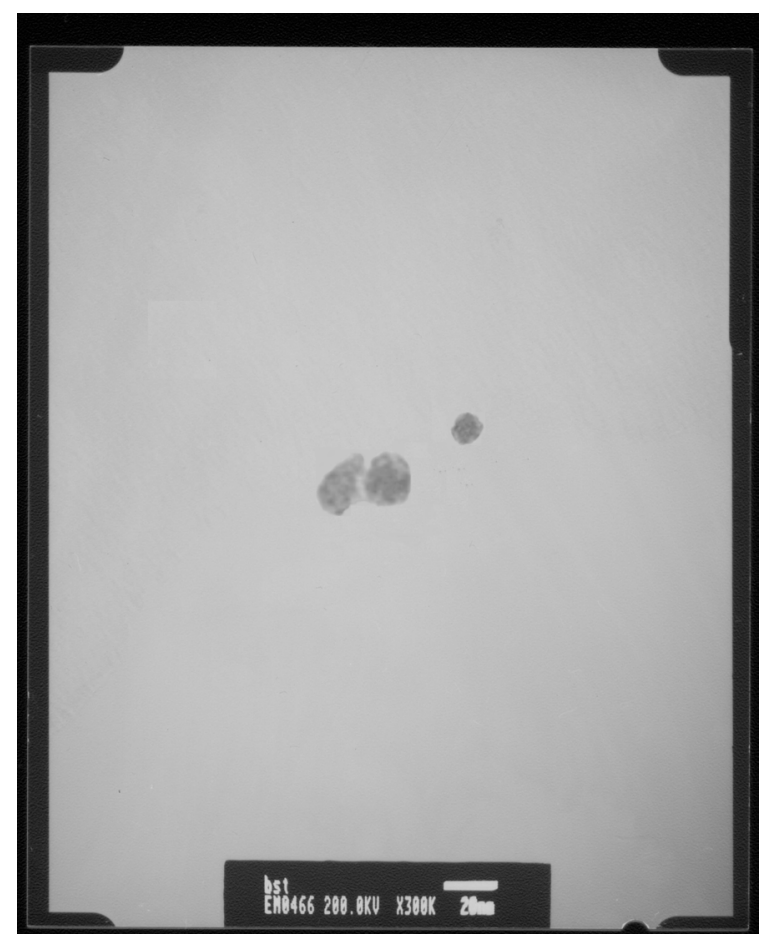

Figure S4. TEM picture of silica-chitosan composite nanoparticles (Scale bar, 20 $\mathrm{nm})$. 